\title{
Associating Sleep Problems With Advanced Cancer Diagnosis, and Immune Checkpoint Treatment Outcomes: a Pilot Study
}

Arthur Sillah ( $\square$ artsil2010@gmail.com )

University of Washington School of Public Health https://orcid.org/0000-0003-1065-6735

Ulrike Peters

Fred Hutchinson Cancer Research Center

Nathaniel F. Watson

University of Washington School of Medicine

Scott S. Tykodi

Fred Hutchinson Cancer Research Center

Evan T. Hall

Fred Hutchinson Cancer Research Center

Allison Silverman

Fred Hutchinson Cancer Research Center

Rachel C Malen

Fred Hutchinson Cancer Research Center

John A. Thompson

Fred Hutchinson Cancer Research Center

Sylvia M. Lee

Fred Hutchinson Cancer Research Center

Shailender Bhatia

Fred Hutchinson Cancer Research Center

Joshua Veatch

Fred Hutchinson Cancer Research Center

Jeannie Warner

Seattle Cancer Care Alliance

Timothy Thornton

University of Washington School of Public Health

Amanda I. Phipps

University of Washington School of Public Health 
Keywords: OSA risk, immune checkpoint inhibitors, metastatic cancer, circadian rhythm

Posted Date: October 18th, 2021

DOI: https://doi.org/10.21203/rs.3.rs-877072/v1

License: (c) (1) This work is licensed under a Creative Commons Attribution 4.0 International License. Read Full License

Version of Record: A version of this preprint was published at Supportive Care in Cancer on January 16th, 2022. See the published version at https://doi.org/10.1007/s00520-022-06825-w. 


\section{Abstract}

Background: Sleep problems (SP) are common in cancer patients but have not been previously assessed in patients receiving immune checkpoint inhibitors (ICI).

Methods: We collected questionnaire data on sleep apnea risk, insomnia and general sleep patterns. We used an adjusted multivariate Poisson regression to calculate prevalence ratios (PRs) and associated 95\% confidence intervals (Cls) for associations between these SP and metastatic versus localized cancer stage (M1 vs M0), and adjusted logistic regression models to calculate ORs for associations between SP with the number of $\mathrm{ICl}$ infusions completed $(6+\mathrm{vs} .<6)$.

Results: Among 32 patients who received ICI treatment, the prevalence of low, intermediate, and high-risk OSA risk was $36 \%, 42 \%$, and $21 \%$, respectively. Overall, $58 \%$ of participants reported clinically significant insomnia. We did not find a significant association between intermediate or high risk OSA (vs. low risk) and metastatic cancer status [PR=1.01 (95\% Cl: 0.28, 3.67)]. Patients in the cohort who reported taking $>15$ minutes to fall asleep were 3.6 times more likely to be diagnosed with metastatic cancer compared to those reporting shorter sleep latency $[95 \% \mathrm{Cl}(1.74,7.35)]$. We did not find a significant association between SP and number of ICl infusions completed.

Conclusion: Our data associating sleep apnea risk, insomnia and sleep patterns with more advanced cancer encourages further exploration in larger-scale observational studies and suggests interventional clinical trials focused on sleep quality improvement could result in better outcomes for these patients.

\section{Introduction}

Sleep problems are a prominent concern of cancer patients. Disturbed sleep is reported by $45-80 \%$ of cancer patients, compared to $29-32 \%$ of the general population. ${ }^{1}$ Sleep problems result from sleep disorders [e.g., sleep-disordered breathing (obstructive sleep apnea (OSA), central sleep apnea, upper airway resistance syndrome), insomnia, narcolepsy], poor sleep quality (e.g., non-restorative sleep, sleep fragmentation), improper sleep timing, irregularity (e.g., constant variation in bedtimes and wake times, frequent random nap episodes), or a non-ideal sleep duration. ${ }^{2}$

Cancer therapy and cancer-related anxiety/stress can cause sleep problems $3^{3,4}$ and/or reflect previously established carcinogenic roles of sleep problems themselves ${ }^{5-7}$ (Fig. 1). The carcinogenic effects of sleep problems could be due to induced intermittent hypoxia $(\mathrm{IH} \text {, a hallmark of OSA })^{8,9}$ and adverse downstream consequences of sleep fragmentation and disruption to the 24-hour circadian rhythm. ${ }^{10,11}$ Mouse models of melanoma and kidney cancers show enhanced tumor growth, invasiveness, and metastasis associated with $\mathrm{IH}_{.} 8,9$ In addition, disruption to normal circadian rhythms increases inflammation and reduces melatonin hormone production, which in both instances promotes cellular damage. $^{10,11}$ 
Increasing evidence shows higher tumor related T cell levels improve prognosis for many types of cancer. ${ }^{12,13}$ Existing and emerging immunotherapies are harnessing this T-cell response to successfully treat several forms of cancer [e.g., melanoma, renal cell carcinoma (RCC), and non-small cell lung cancer (NSCLC)] by inhibiting immune-suppressive proteins such as programmed cell death-1 receptor (PD-1), its ligand PD-L1 (PD-1/PD-L1), and cytotoxic T lymphocyte antigen 4 (CTLA-4). ${ }^{14,15}$ As a result, the US Food and Drug Administration (FDA) has approved seven immune checkpoint inhibitors (ICI) that target CTLA-4 (ipilimumab), PD-1 (nivolumab, pembrolizumab, and cemiplimab), or PD-L1 (atezolizumab, avelumab, and durvalumab) for the treatment of cancer. ${ }^{16}$ Despite the promise of $\mathrm{ICl}$, patient response is not uniformly favorable. Thus, a better understanding of factors predictive of $\mathrm{ICI}$ response is needed. ${ }^{17}$

ICls are increasingly prescribed for late-stage cancer patients. In this context, the oncology field may benefit from new insights into the impact of circadian rhythms (and/or "circadian rhythm disruption") and sleep problems on immune response and cancer aggressiveness in this patient population. Despite the numerous studies of sleep problems in the context of other cancer treatments (e.g., chemotherapy), ${ }^{18,19}$ to date, there have been no studies correlating sleep problems with metastatic versus localized disease or outcomes in cancer patients receiving $\mathrm{ICl}$ therapy. ${ }^{17}$ We report here outcomes from The Lifestyle Attributes and Sleep in Immunotherapy Response (LASIR) Study describing the burden of sleep problems, its relation to the presence of metastases at diagnosis, and its impact on $\mathrm{ICI}$ tolerability in cancer patients previously unexposed to $\mathrm{ICl}$ therapy.

\section{Methods}

\section{Setting}

The LASIR Study was conducted at the Seattle Cancer Care Alliance (SCCA). The SCCA is the clinical practice site for the Cancer Consortium formed by partnership of the Fred Hutchinson Cancer Research Center, the University of Washington, and Seattle Children's Hospital. The SCCA is the only National Cancer Institute-designated comprehensive cancer center serving the five state Washington, Wyoming, Alaska, Montana, and Idaho region. ${ }^{20}$ Based on current clinical practice, which stipulates ICI use for first or second line therapies for advanced kidney cancer ${ }^{21}$ and for some with metastatic melanoma and lung cancers, ${ }^{22}$ the vast majority of study participants had advanced-stage disease (stage III/IV) at ICl initiation.

\section{Participant recruitment}

Patients were study eligible if they were: 1) An adult initiating outpatient treatment with a commercial ICl agent for the first time at the SCCA renal cell carcinoma /melanoma (Ren/Mel) or thoracic / head and neck cancer (THN) clinics, 2) Aged between 18-84 years, 3) Able to provide informed consent and 4) Able to complete the questionnaire in English. Patient recruitment began in April 2019 and was interrupted by the COVID-19 global pandemic in mid-March 2020. Of the 63 eligible patients approached for 
participation, 33 (52\%) enrolled in the study (Fig. 2). Patients received ICI treatment in either the adjuvant (melanoma) or metastatic setting (melanoma, RCC or NSCLC).

\section{Data collection and definitions}

Data was collected from study participants via three sources: a self-administered patient questionnaire, a sleep monitoring device, and electronic medical records.

The questionnaire was self-administered at the time of study enrollment and included an assessment of OSA risk, insomnia, and general sleep patterns. In particular, the primary sleep data was a self-reported 8item validated STOP-BANG questionnaire for the assessment of OSA risk with scores ranging from $0-8$; scores were categorized into low risk (0-2), intermediate risk (3-4), and high risk (5-8) of OSA. Details of the STOP-BANG questionnaire content are described elsewhere. ${ }^{23}$ Secondary sleep data included the 5item Women's Health Initiative Insomnia scale (WHIIS). ${ }^{24}$ The WHIIS requires individuals to rate the quality of their sleep and the frequency with which they experience certain sleep problems in the last month with scores ranging from 0-20 in increasing order of insomnia symptoms; a score $>9$ was considered clinically significant. Details of the WHIIS are described elsewhere. ${ }^{25}$ Other self-reported secondary sleep data included: history of OSA diagnosis, chronotype (e.g., preferred sleep timing over a 24-hour period), sleep latency, and typical sleep duration categorized as shown in Table 4.

Additional relevant lifestyle and patient attribute data collected in the questionnaire included: marital status, educational level, smoking status, and perceived stress. These variables were all categorized as in Table 1. Participants also reported previous diagnoses of hypertension, diabetes, and high cholesterol and treatment status for these conditions to describe their baseline comorbidities. 
Table 1

Selected baseline characteristics overall and according to OSA risk in the LASIR cohort, $n=33$

Sleep apnea risk

\begin{tabular}{|c|c|c|c|}
\hline & $\begin{array}{l}\text { Total }(n= \\
\text { 33) }\end{array}$ & $\begin{array}{l}\text { Low }(n= \\
12)\end{array}$ & $\begin{array}{l}\text { Intermediate/High }(\mathrm{n}= \\
\text { 21) }\end{array}$ \\
\hline Mean age at enrollment (SD) & $61.1(13.4)$ & $58.4(13.9)$ & $62.5(13.1)$ \\
\hline Males & 60.6 & 16.7 & 85.7 \\
\hline married, domestic partnered & 63.6 & 50.0 & 71.4 \\
\hline \multicolumn{4}{|l|}{ Education } \\
\hline$<$ college & 30.3 & 25.0 & 33.3 \\
\hline College degree & 45.5 & 66.7 & 33.3 \\
\hline Grad or professional deg & 24.2 & 8.3 & 33.3 \\
\hline White & 84.8 & 83.3 & 84.7 \\
\hline Hispanic & 3.3 & 0 & 4.8 \\
\hline \multicolumn{4}{|l|}{ smoking at cancer diagnosis } \\
\hline Current & 3.3 & 0 & 4.8 \\
\hline Former & 45.5 & 33.3 & 52.4 \\
\hline Never & 51.5 & 66.7 & 42.9 \\
\hline BMl at cancer $\mathrm{dx} \mathrm{kg} / \mathrm{m} 2$ mean (SD) & $30.9(8.1)$ & $29.8(11.4)$ & $31.4(5.7)$ \\
\hline$<25$ & 21.2 & 41.7 & 9.5 \\
\hline $25-29$ & 30.3 & 16.7 & 38.1 \\
\hline $30+$ & 48.5 & 41.7 & 52.4 \\
\hline \multicolumn{4}{|l|}{ Perceived stress } \\
\hline median (IQR) & $\begin{array}{l}5.0(3.0 \\
6.0)\end{array}$ & $\begin{array}{l}5.0(2.5 \\
6.0)\end{array}$ & $6.0(3.0,7.0)$ \\
\hline Low stress $(<8)$ & 81.8 & 83.3 & 81 \\
\hline High stress (8-16) & 18.2 & 16.7 & 19.1 \\
\hline \multicolumn{4}{|l|}{ Self-reported disease history/ medication } \\
\hline $\begin{array}{l}\text { High blood pressure/ hypertension } \\
\text { medications }\end{array}$ & 48.5 & 25.0 & 61.9 \\
\hline high cholesterol/ Cholesterol medications & 39.4 & 25.0 & 47.6 \\
\hline
\end{tabular}




\section{Sleep apnea risk}

Diabetes/ treated diabetes

9.1

0.0

14.3

Categorical variables are in percentages, continuous measures in mean (SD: Standard Deviation) or median (IQR: Inter quartile range), *\% may not sum to $100 \%$ due to missing data or rounding

The Sleepscore Max ${ }^{26}$ bedside sleep monitoring device was used to measure nighttime sleep patterns for a maximum of 30 days post ICl initiation in a subset of consented participants $(n=12)$. The Sleepscore Max is a non-contact sleep sensor which uses a smartphone app and web-based app to record sleep patterns. ${ }^{26}$ Specifically, it uses smartphone speaker capabilities to track and measure breathing rate and body movement, to provide an in-depth analysis of sleep, including chest and abdominal respiratory movement, to measure key sleep attributes. ${ }^{26}$ The device recorded data on total night sleep time, sleep onset latency, wake after sleep onset, number of awakenings, sleep architecture (deep, light and rapid eyemovement (REM) sleep) and overall sleep quality (Sleep score, higher score indicating better sleep quality).

Electronic health records (EHR) data was collected six months post ICl initiation. Extracted EHR data included age, gender, weight, and height on the date corresponding to the patient's last visit at SCCA prior to or at ICl initiation. Body mass index (BMI) was calculated by dividing weight in kilograms by height in meters squared and grouped into three categories: $<25 \mathrm{~kg} / \mathrm{m}^{2}, 25-29 \mathrm{~kg} / \mathrm{m}^{2}$, and $\geq 30 \mathrm{~kg} / \mathrm{m}^{2}$. We extracted information pertaining to cancer diagnosis date, tumor attributes at diagnosis, and prior cancer treatments (chemotherapy, radiotherapy, surgery and other treatment regimens). Tumor attributes included M-stage and summary stage (IIV) at diagnosis. We extracted dates of the first six, and last ICl infusions along with the type of each $\mathrm{ICl}$ initiated, and $\mathrm{ICl}$ adverse events incidence within the first six months of ICl initiation. We extracted participant vital status (death status, cause of death, and date of death) within the first six months of $\mathrm{ICl}$ initiation.

\section{Main outcomes definitions}

Metastatic cancer status was defined by M-stage (M0 vs. M1). ICl tolerance was defined in close consultation with experienced SCCA oncologists. Six or more infusions were considered to represent both $\mathrm{ICI}$ tolerability and treatment benefit within the six-month assessment period.

\section{Statistical analysis}

All analytical procedures were conducted using Stata 14.0 (College Station, Texas) ${ }^{27}$, with statistical significance considered at a 2-sided alpha value of 0.05 . In descriptive analyses, we examined the distribution of participants' baseline characteristics overall and stratified by OSA risk (low vs. intermediate/high). We ran a multivariate Poisson regression with robust standard errors (SEs) to assess the association between sleep problems and metastatic tumor status at diagnosis [M-stage (M0 vs. M1)], 
adjusting for age, gender, and BMI. We opted to use a Poisson model instead of a logistic model due to sleep problems being common in cancer populations and the high prevalence of advanced disease in cancer patients initiating ICl. We used robust SEs in the Poisson model to account for any violation of the distribution assumption that the variance equals the mean. We reported the prevalence ratio (PR) and associated $95 \% \mathrm{Cl}$ for associations.

We used logistic regression models to calculate odds ratios (ORs) to assess the associations of OSA and insomnia risk, chronotype and sleep latency with the number of $\mathrm{ICl}$ infusions $(6+v s<6)$, adjusting for the following selected attributes: male gender, age at ICI treatment, and prior cancer treatment.

\section{Results}

\section{Patient characteristics}

The mean age of the cohort was 61 years, $61 \%$ were male, $85 \%$ were white, $64 \%$ were partnered, $70 \%$ had a college degree, $49 \%$ had BMl greater than $30 \mathrm{~kg} / \mathrm{m}^{2}$, and $3 \%$ were current smokers. Patients with intermediate or high risk OSA were, on average, older and had: 1) fewer years of education, 2) high stress levels, and 3) higher prevalence of diabetes, high cholesterol, and high blood pressure. (Table 1)

\section{Tumor attributes}

The most common cancer site was melanoma (52\%). Seventy three percent were diagnosed with a latestage disease (III/IV), and $42 \%$ with a metastatic disease. The majority of patients had had cancer treatment prior to ICl initiation (64\%), of whom $66.7 \%$ had surgery, $23.8 \%$ were treated with chemotherapy, and $19 \%$ with radiotherapy. (Table 2 ) 
Table 2

Selected baseline cancer attributes in the LASIR cohort, $n=$ 33

\begin{tabular}{|ll|}
\hline & Total $(\mathbf{n}=\mathbf{3 3})$ \\
\hline Cancer site & \\
\hline Melanoma & 51.5 \\
\hline Squamous Cell Carcinoma & 6.1 \\
\hline Renal Cell Carcinoma & 21.2 \\
\hline Lung & 21.2 \\
\hline Cancer summary stage at diagnosis & \\
\hline I & 9.1 \\
\hline II & 9.1 \\
\hline III & 33.3 \\
\hline IV & 39.4 \\
\hline Cancer treatment prior to ICl & 63.6 \\
\hline Chemotherapy & 23.8 \\
\hline Radiotherapy & 19.1 \\
\hline Surgery & 66.7 \\
\hline Gene therapy & 15.3 \\
\hline *\% may not sum to 100\% due to missing data or rounding \\
\hline SD: Standard Deviation & \\
\hline
\end{tabular}

\section{ICl treatment attributes}

The majority of patients initiated a PD-1 blockade (94\%), $16 \%$ initiated ICI with chemotherapy, and $72 \%$ had six or more $\mathrm{ICl}$ infusions within a six-month period. Participants reported, on average, three adverse events post ICI initiation. The most common incident adverse events were rash and vitiligo (47\%), general body pain (39\%), hypothyroidism (25\%) and severe diarrhea (22\%). Thirteen percent of enrolled participants died within the six-month follow-up period. The average time from cancer diagnosis to death and from ICl initiation to death was 13 months and two months, respectively. (Table 3 ) 
Table 3

Distribution of ICl attributes in the LASIR Cohort, $\mathrm{N}=32$ *

\begin{tabular}{|c|c|}
\hline & Total $(n=32)$ \\
\hline \multicolumn{2}{|l|}{ Months from cancer diagnosis to ICl, Mean (SD) } \\
\hline \multicolumn{2}{|l|}{$\mathrm{ICl}$ initiated } \\
\hline Cemiplimab (PD-1) & 6.3 \\
\hline Ipilimumab (CTLA-4) & 6.3 \\
\hline Nivolumab (PD-1) & 43.8 \\
\hline Pembrolizumab (PD-1) & 43.8 \\
\hline ICl initiated with chemo & 16.1 \\
\hline Years from cancer $\mathrm{dx}$ to ICl, median (IQR) & $0.4(0.2,2.3)$ \\
\hline Total ICl initiation mean (SD) & $6.8(3.6)$ \\
\hline$\%<6$ infusions & 28.2 \\
\hline$\% 6+$ infusions & 71.9 \\
\hline Time between 1st and last Infusion, months (SD) & $4.00(2.08)$ \\
\hline \multicolumn{2}{|l|}{ \% Incidence of Common Adverse Events } \\
\hline Rash \& Vitiligo & 46.9 \\
\hline General body pain & 39.3 \\
\hline Hypothyroidism & 25.0 \\
\hline Severe Diarrhea & 22.0 \\
\hline Total events, mean (SD) & $3.3(2.5)$ \\
\hline \multicolumn{2}{|l|}{ Vital Status } \\
\hline$\%$ Death * & 12.5 \\
\hline Time from cancer $\mathrm{dx}$ to death, months mean (SD) & $13.2(7.7,39.8)$ \\
\hline Time from ICl to death, months mean (SD) & $2.1(1.32,5.2)$ \\
\hline SD: Standard Deviation; * 1 patient died without $g$ & ing $\mathrm{ICl}$ \\
\hline
\end{tabular}

Prevalence of sleep problems 
The prevalence of OSA symptoms was $21 \%$ for daytime sleepiness, $33 \%$ for observed apnea, and $21 \%$ for snoring; $51 \%$ reported none of these three symptoms. Among those with sleep apnea symptoms, the prevalence of low, intermediate, and high OSA risk was $36 \%, 42 \%$, and $21 \%$ respectively. The prevalence of a self-reported OSA diagnosis at enrollment was $18 \%$. The prevalence of low, intermediate, and high OSA risk among those who self-reported an OSA diagnosis was $33 \%, 17 \%$ and $50 \%$ respectively.

Of the secondary sleep problems considered, $58 \%$ of participants reported clinically significant insomnia, $72 \%$ experienced average or restless sleep, 30\% reported taking longer than $15 \mathrm{~min}$ to fall asleep, $44 \%$ had non-ideal night sleep. Thirty six percent of participants reported an evening chronotype. (Table 4) 
Prevalence ratios (PRs) for tumor aggressiveness comparing across sleep problem groups in the LASIR cohort, $\mathrm{N}=33$

Diagnosis M-stage [M0 ( $n=19)$ vs M1 (n-14)]

\section{Summary* PR $(95 \% \mathrm{Cl}) \quad$ p-values}

\section{Primary Sleep problems}

\section{Sleep apnea Risk (0-8)}

Total score (1-unit increment)

$3.2(1.7) \quad 1.15(0.74,1.77)$

0.533

Intermediate risk (3-4)/High risk (5-8)

$42.4 / 21.2 \quad 1.01(0.28,3.67)$

0.990

low risk (0-2) (ref)

36.4

1

\section{Secondary sleep problems}

Insomnia Risk (0-20)

Total score (1-unit increment)

$9.5(4.3)$

$1.08(0.97,1.20)$

0.147

Clinically significant insomnia (9+)

57.6

$1.24(0.49,3.14)$

0.649

Not clinically significant $(<9)$ (ref)

42.4

1

\section{Sleep latency, min}

$15+$

0-14 (ref)

Total sleep duration, $\mathrm{h}$

$<=6$ or $9+$

7-8 (ref)

\section{Chronotype}

Evening

Morning (ref)

\section{Overall sleep quality}

\begin{tabular}{llll} 
Restless & 33.3 & $2.25(0.51,9.91)$ & 0.285 \\
\hline Average & 39.4 & $1.31(0.40,4.28)$ & 0.659 \\
\hline Sound (ref) & 27.3 & 1 & \\
\hline M-stage - Metastatic cancer stage & & & \\
\hline Adjusted for age, male, body mass index. & &
\end{tabular}


*Summary: Categorical variable in \%; continuous variables in mean (SD)

*\% may not sum up to $100 \%$ due to missing data or rounding

The SleepScore Max data is summarized in Supplemental Fig. 1 and Supplemental Table 1. In this small sample, there was little evidence of changes in within-person sleep patterns over the 30 days period post $\mathrm{ICl}$ initiation. Overall, the mean nighttime total sleep duration was 6 hours, mean latency was 19 min, mean number of wake times was five. Participants had an average of four hours of light, one hour of deep and REM sleep. The sleep quality overall measured by the Sleepscore Max devices was $80 \%$.

\section{Association between sleep problems and metastatic cancer}

Table 4 presents PRs and 95\% Cls for associations between sleep problems and metastatic cancer at diagnosis. We did not find a significant association between intermediate or high risk OSA and metastatic cancer compared to low risk OSA [1.01 $(0.28,3.67)]$; similarly, metastatic status was not associated with continuous STOP-BANG scores for OSA risk $[1.15(0.74,1.77)]$.

Of the secondary sleep attributes considered, patients reporting more than 15 minutes to fall asleep were 3.6 times more likely to have been diagnosed with metastatic cancer compared to those reporting shorter sleep latency $[95 \% \mathrm{Cl}(1.74,7.35)]$. Additionally, patients reporting a evening chronotype were more likely to have been diagnosed with metastatic cancer compared to those reporting morning chronotypes [4.36 $(1.73,11.00)]$.

\section{Association between sleep problems and ICI treatment tolerance}

Table 5 presents HRs and 95\% Cls for associations between sleep problems and the number of ICI infusions as a measurement of $\mathrm{ICl}$ treatment tolerance. We did not find any significant association between intermediate or high risk OSA and six or more infusions compared to low risk OSA [0.27 (0.02, $3.41)]$ and between continuous OSA risk scores and six or more infusions $[0.72(0.37,1.40)]$. Similarly, we found no significant association between insomnia and six or more infusions $[0.23(0.03,1.60)]$ and between insomnia total scores and six or more infusions $[0.77(0.59,1.02)]$. Additionally, we did not find any significant association between evening chronotype and six or more infusions compared to morning chronotype $[0.57(0.11,2.97)]$ and patients reporting more than 15 minutes to fall asleep compared to those reporting shorter sleep latency $[0.51(0.07,3.53)]$. 
Table 5

Association between sleep problems and number of ICI infusions in the LASIR Cohort, $N=32$

Number of infusions $(6+v s<6)$

Tumor attributes

OR $(95 \% \mathrm{Cl})$

p-values

Sleep apnea risk (STOP-BANG, 0-8)

Continuous

$0.72(0.37,1.39)$

0.335

Total score (1-unit increment)

Categorical

$0.27(0.02,3.41)$

0.308

Intermediate risk (3-4)/High risk (5-8)

low risk (0-2) (reference)

1

Insomnia Risk (WHIIS, 0-20)

Continuous

$0.77(0.59,1.02)$

0.071

Total score (1-unit increment)

\section{Categorical}

$0.23(0.03,1.60)$

0.138

Clinically significant insomnia (9+)

Not clinically significant $<9$ (reference)

1

\section{Chronotype}

Evening

$0.57(0.11,2.97)$

0.506

Morning (ref)

1

Sleep latency, min

$15+$

$0.51(0.07,3.53)$

0.493

$0-14$ (ref)

WHIIS: Health Initiative Insomnia scale

STOP-BANG: Snoring, daytime Tiredness, observed apnea, Blood Pressure, Body Mass Index, Age, Neck circumference and Gender

Treatment tolerability: Number of infusions $>6$

Adjusted: age, sex, prior cancer treatment

\section{Discussion}

This study is the first to examine sleep patterns in cancer patients receiving ICI therapy. Our study also assessed objective sleep patterns over time in cancer patients post ICI treatment initiation, which, to our 
knowledge, has not been done before. We found a high burden of sleep problems in this ICl cohort. In particular, two-thirds of enrolled participants had intermediate to high sleep apnea risk and an average / restless night sleep, more than half experienced clinically significant insomnia, and about a third reported taking longer than $15 \mathrm{~min}$ to fall asleep and evening chronotype. We also observed that objective sleep patterns remained mostly consistent over time in a subset of participants assessed, suggesting that sleep information collected at study baseline remains indicative of sleep patterns through, at least, early stages of the treatment period. In a multivariable-adjusted regression analysis, we did not find a statistically significant association between intermediate or high risk OSA and metastatic cancer compared to low risk OSA. However, of the secondary sleep problems assessed, patients who reported taking longer to fall asleep were more likely to have been diagnosed with metastatic cancer compared to those reporting shorter sleep latency. Additionally, patients reporting an evening chronotype (patients who are most active and alert in the evening) were more likely to have been diagnosed with metastatic cancer compared to those reporting a morning chronotype (patients who are most active and alert in the morning). Our second goal was to determine the association between sleep problems and ICI treatment tolerance. While we did not find any significant association between OSA risk, insomnia, and six or more infusions during the first six months after ICl initiation, the direction of the estimates showed higher odds for poor ICI treatment tolerance in patients with certain sleep problems.

Our study corroborates other studies of sleep problems in more traditional cancer treatment cohorts (e.g., patients receiving radiotherapy and chemotherapy). ${ }^{18,19}$ In particular, prior studies noted a high burden of sleep problems, including insufficient sleep duration, insomnia symptoms, and poor overall sleep quality, ${ }^{18,19}$ that have been, in turn, linked with poor cancer prognosis. ${ }^{1,28}$ For instance, cancer patients with insufficient sleep duration ( $\leq 6 \mathrm{~h}$ sleep/night) and who snore might be experiencing more severe underlying sleep problems and, therefore, subsequent worse cancer outcomes. ${ }^{1,28}$ Additionally, the longitudinal sleep data collected with the sleep score max may suggest the impact of the single time point sleep are durable over time.

The results from these analyses have some key limitations. Chief amongst them is the limited sample size, which could explain the mostly non-statistically significant results. Relatedly, although we limited our study to patients receiving $\mathrm{ICl}$ within two clinical units at a single institution, the physiologic insults of sleep problems on cancer prognosis are heterogeneous across cancer sites and possibly molecular types. ${ }^{17,29,30}$ Thus, by combining data across patients with multiple cancer sites, some cancer sitespecific relationships may have been obscured. However, small numbers precluded us from conducting site-specific analyses.

Secondly, study participants self-reported their sleep problems. Given the focus of this study on the patient population initiating $\mathrm{ICl}$ for the treatment of their late-stage cancer, several factors might be impacting participant sleep patterns (e.g., stress, side effects from previous lines of therapy as illustrated in Fig. 1); thus, observed sleep patterns may not be reflective of pre-diagnostic sleep patterns. In addition, it is possible that poor cancer prognosis could cause sleep problems instead of the reverse (Fig. 1). However, this is less concerning for the $\mathrm{ICl}$ response outcome analysis since it is downstream of reported 
sleep problems at enrollment. Additionally, our study did not measure immune response biomarkers (e.g., inflammatory markers cytokines, including IL-1, IL-6, and TNF-a) that may be more sensitive to underlying sleep problems. ${ }^{31,32}$ However, our sleep data is based on validated questionnaires and, unlike most studies assessing sleep problems in cancer which have focused on a single sleep dimension (e.g., sleep duration) ${ }^{33}$, we evaluated multiple sleep dimensions, including STOP-BANG OSA risk levels. ${ }^{23,34}$

Another important study limitation is in the assessment of $\mathrm{ICI}$ response. The primary response measure in $\mathrm{ICl}$ studies and the clinic settings is based on RECIST 1.1 guidelines. ${ }^{35}$ The guideline is based on tumor imaging data incorporating information on changes in lesion size and new lesions to distinguish $\mathrm{ICl}$ "responders" from "non-responders". ${ }^{35}$ We were unable to incorporate the guidelines into our study due to lack of this data at the end of 6 months follow-up.

Finally, there is also an issue of representativeness of the SCCA cancer population to the general ICI cancer treatment population. Specifically, our study population is racially homogenous (mainly of European descent, $85 \%$ white) and likely have higher socio-economic status.

Despite these study limitations, this study is the first, to our knowledge, to examine the biologically plausible and potential impact of sleep problems in cancer patients receiving ICI therapy. In result, given the burden and potential impact of sleep problems on ICI treatment response, we believe the study limitations are outweighed by the importance of this study in setting the stage for larger studies with more comprehensive sleep and $\mathrm{ICI}$ response assessments.

\section{Conclusions}

This study provides new insights into the burden of sleep problems on cancer patients receiving ICI treatment. We hope these results will motivate larger studies of ICl-treated patients to include sleep problems in their assessment ${ }^{17}$ that could potentially inform interventional clinical trials focused on sleep quality improvement in ICl treatment populations.

\section{Declarations}

\section{Funding:}

This work was supported by National Institutes of Health (NIH) grants T32CA094880, and by pilot funding provided by the Public Health Sciences Division and Immunotherapy Integrated Research Center at the Fred Hutchinson Cancer Research Center.

\section{Conflicts of interest:}

SB: reports advisory board participation (with honorarium) from Genentech, EMD-Serono, Bristol-MyersSquibb (BMS) and Sanofi-Genzyme; and research funding to his institution (University of Washington) 
from EMD-Serono, Merck, BMS, Novartis, Incyte, Exicure, Nektar, NantKwest, Oncosec and Immune Design. All other authors declare there is no conflict of interest.

\section{Availability of data and material:}

Not applicable

\section{Code availability:}

Not applicable

\section{Authors' contribution:}

Arthur Sillah conceptualized and codeveloped and implemented the study, data collection, analysis, and writing. Alison Silverman co-implemented the study, data collection and general study management along with Rachel Malen. Amanda Phipps, Ulrike Peters, Nathaniel Watson did general work supervision and writing. All other Authors contributed content knowledge, writing and extensive edits and feedback.

\section{Ethics approval:}

The study protocol was reviewed and approved by the Fred hutch cancer research center, Seattle WA USA.

\section{Consent to participate:}

All included study participants signed an informed consent

\section{Consent for publication:}

All authors give their consent for publication.

\section{References}

1. Collins KP, Geller DA, Antoni M, et al. Sleep duration is associated with survival in advanced cancer patients. Sleep Med. 2017;32:208-212. doi:S1389-9457(17)30013-8 [pii]

2. Santamaria-Martos F, Sánchez-de-la-Torre M, Martínez-García MA. Sleep and Cancer: Clinical Studies and Opportunities for Personalized Medicine. Curr Sleep Med Rep. 2017;3(1):11-21.

3. Dickerson SS, Connors LM, Fayad A, Dean GE. Sleep-wake disturbances in cancer patients: narrative review of literature focusing on improving quality of life outcomes. Nat Sci Sleep. 2014;6:85-100. doi:10.2147/NSS.S34846 [doi]

4. Wu HS, Harden JK. Symptom burden and quality of life in survivorship: a review of the literature. Cancer Nurs. 2015;38(1):E29-54. doi:10.1097/NCC.0000000000000135 [doi]

5. Dycke KCV, Rodenburg W, Oostrom CT van, et al. Chronically alternating light cycles increase breast cancer risk in mice. Curr Biol. 2015;25(14):1932-1937. 
6. Hansen J, Stevens RG. Case-control study of shift-work and breast cancer risk in Danish nurses: impact of shift systems. Eur J Cancer Oxf Engl 1990. 2012;48(11):1722-1729. doi:10.1016/j.ejca.2011.07.005 [doi]

7. ljaz S, Verbeek J, Seidler A, et al. Night-shift work and breast cancer-a systematic review and metaanalysis. Scand J Work Environ Health. Published online 2013:431-447.

8. Vilaseca A, Campillo N, Torres M, et al. Intermittent hypoxia increases kidney tumor vascularization in a murine model of sleep apnea. PloS One. 2017;12(6):e0179444.

9. Almendros I, Martinez-Garcia MA, Farré R, Gozal D. Obesity, sleep apnea, and cancer. Int J Obes. Published online 2020:1-15.

10. Cherrie JW, Crawford JO, Davis A, et al. A review of the impact of shift-work on cancer: summary of the evidence for practitioners. Policy Pract Health Saf. Published online 2017:1-7.

11. Nobis CC, Labrecque N, Cermakian N. Circadian control of antigen-specific T cell responses. ChronoPhysiology Ther. 2016;6:65-74.

12. Tumeh PC, Harview CL, Yearley JH, et al. PD-1 blockade induces responses by inhibiting adaptive immune resistance. Nature. 2014;515(7528):568.

13. Hu G, Wang S. Tumor-infiltrating CD45RO Memory T Lymphocytes Predict Favorable Clinical Outcome in Solid Tumors. Sci Rep. 2017;7(1):10376.

14. Iwai Y, Hamanishi J, Chamoto K, Honjo T. Cancer immunotherapies targeting the PD-1 signaling pathway. J Biomed Sci. 2017;24(1):26.

15. Topalian SL, Taube JM, Anders RA, Pardoll DM. Mechanism-driven biomarkers to guide immune checkpoint blockade in cancer therapy. Nat Rev Cancer. 2016;16(5):275.

16. Food, INFORMATION DA-HOP.

https://www.accessdata.fda.gov/drugsatfda_docs/label/2018/761097s000lbl.pdf accessed $5 / 16 / 2020$.

17. Sillah A, Tykodi SS, Hall ET, et al. Predict Lifestyle Markers Effic Cancer Immune Checkp Inhib Comment. Published online 2020.

18. Ancoli-Israel S. Sleep disturbances in cancer: a review. Sleep Med Res. 2015;6(2):45-49.

19. Chen M-L, Yu C-T, Yang C-H. Sleep disturbances and quality of life in lung cancer patients undergoing chemotherapy. Lung Cancer. 2008;62(3):391-400.

20. Alliance SCC. http://immunotherapy.seattlecca.org/ , Accessed 4.30.2018.

21. Rini Bl, McDermott DF, Hammers H, et al. Society for Immunotherapy of Cancer consensus statement on immunotherapy for the treatment of renal cell carcinoma. J Immunother Cancer. 2016;4(1):81.

22. Kaufman HL, Kirkwood JM, Hodi FS, et al. The Society for Immunotherapy of Cancer consensus statement on tumour immunotherapy for the treatment of cutaneous melanoma. Nat Rev Clin Oncol. 2013;10(10):588.

23. Chung F, Abdullah H, Liao P. STOP BANG Questionnaire. Chest. 2016;149(4):631-638. 
24. Levine DW, Lewis MA, Bowen DJ, et al. Reliability and validity of Women's Health Initiative Insomnia Rating Scale. Psychol Assess. 2003;15(2):137.

25. Bardwell WA, Profant J, Casden DR, et al. The relative importance of specific risk factors for insomnia in women treated for early-stage breast cancer. Psycho-Oncology. 2008;17(1):9-18.

26. Resmed. https://www.sleepscore.com/sleepscore-max-sleep-trackeraccessed 05.01.2018.

27. StataCorp. Stata Statistical Software: Release 14. College Station, TX: StataCorp LP. Published online 2015.

28. Phipps Al, Bhatti P, Neuhouser ML, et al. Pre-diagnostic Sleep Duration and Sleep Quality in Relation to Subsequent Cancer Survival. J Clin Sleep Med JCSM Off Publ Am Acad Sleep Med. 2016;12(4):495-503. doi:10.5664/jcsm.5674 [doi]

29. Gozal D, Ham SA, Mokhlesi B. Sleep Apnea and Cancer: Analysis of a Nationwide Population Sample. Sleep. 2016;39(8):1493-1500. doi:10.5665/sleep.6004 [doi]

30. Marhuenda E, Campillo N, Gabasa M, et al. Effects of Sustained and Intermittent Hypoxia on Human Lung Cancer Cells. Am J Respir Cell Mol Biol. 2019;61(4):540-544.

31. Montesi SB, Bajwa EK, Malhotra A. Biomarkers of sleep apnea. Chest. 2012;142(1):239-245.

32. Clinton JM, Davis CJ, Zielinski MR, Jewett KA, Krueger JM. Biochemical regulation of sleep and sleep biomarkers. J Clin Sleep Med. 2011;7(05):S38-S42.

33. Otte JL, Carpenter JS, Manchanda S, et al. Systematic review of sleep disorders in cancer patients: can the prevalence of sleep disorders be ascertained? Cancer Med. 2015;4(2):183-200.

34. Chung F, Subramanyam R, Liao P, Sasaki E, Shapiro C, Sun Y. High STOP-Bang score indicates a high probability of obstructive sleep apnoea. Br J Anaesth. 2012;108(5):768-775.

35. Eisenhauer EA, Therasse P, Bogaerts $\mathrm{J}$, et al. New response evaluation criteria in solid tumours: revised RECIST guideline (version 1.1). Eur J Cancer. 2009;45(2):228-247.

\section{Figures}




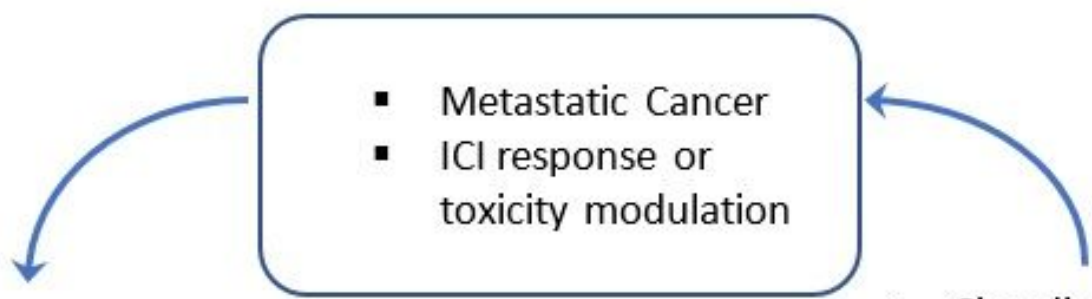

- Circadian disruption

- Cancer symptoms

- Stress and Anxiety

- $\downarrow$ Melatonin

- Treatment side effects

- Modulation of immune responses

- Sleep apnea induced Intermittent Hypoxia.

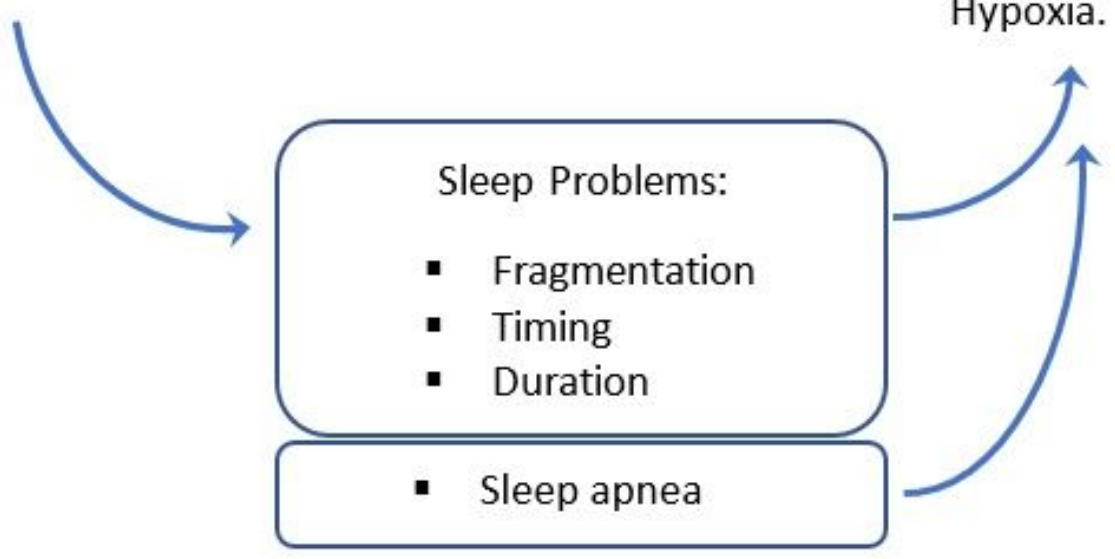

\section{Figure 1}

Conceptual model linking sleep problems to tumor aggressiveness, cancer symptoms and side effects, and Immune Checkpoint Inhibitor (ICI) outcomes 


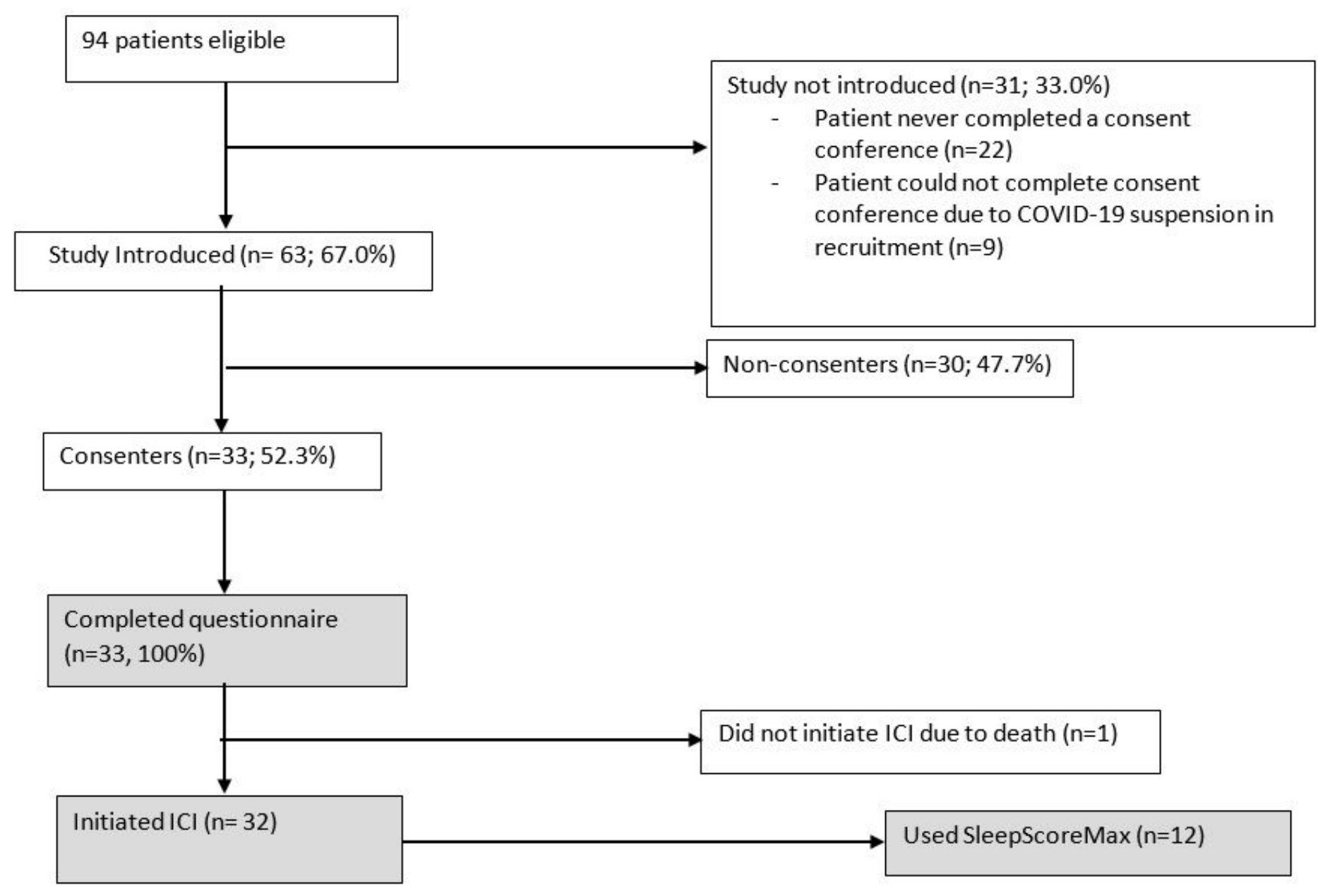

Figure 2

LASIR Study recruitment and retention

\section{Supplementary Files}

This is a list of supplementary files associated with this preprint. Click to download.

- SupplementaryData.docx 